\title{
REVISÃO DE LITERATURA SOBRE O USO DA REALIDADE AUMENTADA NO ENSINO DE QUÍMICA
}

\author{
Alex Eder da Rocha Mazzuco - UFRGS - alexmazzuco@gmail.com - 0000-0002-4888-7005 \\ Aliane Loureiro Krassmann - IFFar - alkrassmann@gmail.com - 0000-0001-7553-5518 \\ Ederson Bastiani - UFRGS - edersonbastiani@gmail.com - 0000-0002-2830-6568 \\ Eliseo Berni Reategui - UFRGS - eliseoreategui@gmail.com - 0000-0002-5025-9710
}

RESUMO: Nos últimos anos a Realidade Aumentada (RA) tem atraído a atenção de pesquisadores de diversas áreas. Por sua especial capacidade de criar ambientes que facilitam a compreensão de conceitos abstratos, esta tecnologia tem sido amplamente utilizada no ensino de Química. Neste contexto, este trabalho apresenta uma revisão sistemática de literatura, buscando revelar as tendências científicas, o público-alvo e os principais tópicos em que essa tecnologia é aplicada. Foram revisados artigos publicados em periódicos e conferências internacionais no período de 2011 a 2020. Entre os principais resultados encontrados, está o constante aumento de estudos nos últimos três anos, o público-alvo sendo formado majoritariamente por estudantes do ensino superior, e estruturas moleculares como o principal tópico da Química em que a RA é utilizada.

Palavras-chave: Realidade Aumentada, Ensino de Química, Tecnologia Educacional.

\section{LITERATURE REVIEW ON THE USE OF AUGMENTED REALITY IN CHEMISTRY TEACHING}

\begin{abstract}
In recent years, Augmented Reality (AR) has attracted the attention of researchers from different areas. Due to its special ability to create environments that facilitate the understanding of abstract concepts, this technology has been widely used in the teaching of Chemistry. In this context, this work presents a systematic literature review, seeking to reveal scientific trends, the target audience and the main topics in which this technology is applied. Articles published in international journals and conferences from 2011 to 2020 were reviewed. Among the main results found, there is the constant increase in studies in the last three years, the target audience being formed mostly by higher education students, and molecular structures such as the main topic of Chemistry in which AR is used.
\end{abstract}

Keywords: Augmented Reality, Chemistry Teaching, Educational Technology.

\section{Introdução}

A Realidade Aumentada (RA) é uma tecnologia que consiste na sobreposição contínua de imagens virtuais geradas por computador no mundo real, ampliando, assim, o acesso à informação, gerando novas oportunidades de interação (AZUMA, 1997). Desde o início da década de 1960, com o crescente desenvolvimento tecnológico, hardware e software tornaram-se mais avançados, fazendo com que os dispositivos modernos suportem, adequadamente, aplicações que demandam uma quantidade significativa de recursos computacionais, como a RA (HANAFI et al., 2019).

Este cenário levou a um rápido aumento na adoção da tecnologia de RA por inúmeros campos, como pela indústria e comércio (BLANCO-NOVOA et al., 2018), pela medicina (GSAXNER et al., 2021) e pelo turismo (HAN et al., 2021). No entanto, V. $19 \mathrm{~N}^{\circ} 1$, julho, 2021 DOI: https://doi.org/10.22456/1679-1916.118530 RENOTE 
embora todas as áreas do conhecimento possam potencialmente serem beneficiadas pela RA, a educação, de acordo com Da Silva et al. (2019), é particularmente mais suscetível a modificações por meio da inserção dessa tecnologia. Essas mudanças estão essencialmente relacionadas à capacidade da RA conceber ambientes de convivência entre realidade e virtualidade, permitindo que os alunos vivenciem fenômenos, que de outras formas seriam impossíveis de serem experimentados.

Nos últimos anos, a área da educação tem demonstrado um crescente interesse pela tecnologia de RA (ZHANG et al., 2021), estando presente em diversos níveis de ensino, da pré-escola à pós-graduação. Inúmeros benefícios são apontados quando a RA é utilizada como ferramenta de apoio às metodologias de ensino (PRIBEANU et al., 2017). Ela oportuniza a educadores o fornecimento de novas formas de apresentação de materiais de aprendizagem, e a estudantes a possibilidade de interagirem espontaneamente com esses materiais (CAI et al., 2021). Permitindo, também, que o processo de percepção e de memorização do conteúdo educacional seja simplificado, aprimorando a habilidade de raciocínio espacial e abstrato (KONONOVA et al., 2019).

Processos pedagógicos de diferentes domínios do conhecimento estão gradativamente sendo complementados por estratégias que envolvem a RA. Kerr e Lawson (2020), por exemplo, descreveram o desenvolvimento de um protótipo de RA, direcionado ao ensino de princípios fundamentais da Arquitetura Paisagística. Önal e Önal (2021), por sua vez, utilizaram a RA no ensino de Astronomia, e Celik et al. (2020) projetaram e desenvolveram um aplicativo de RA para auxiliar no ensino de Biologia, contemplando interações com a estrutura anatômica do coração. Inúmeras pesquisas envolvendo a RA também estão sendo realizadas em disciplinas como História (AZRINA et al., 2021), Geografia (ADEDOKUN-SHITTU et al., 2020), Matemática (VELÁZQUEZ e MÉNDEZ, 2021), Física (CAI et al., 2021) e Química (ZHANG et al., 2021).

No ensino de Química, a dificuldade de abstração para compreender seus conceitos pode ser atribuída à necessidade de associações entre as três dimensões que envolvem o conhecimento desta ciência: submicroscópicas, simbólicas e macroscópicas (NICHELE et al., 2020). Esses conceitos envolvem tópicos complexos, como "Estruturas Moleculares", "Ligações Químicas", "Geometria Molecular" e "Quiralidade Molecular". Nesse sentido, a RA tem se mostrado uma ferramenta significativa para o ensino de Química, principalmente, segundo Syawaludin et al. (2019), por ser um recurso facilitador do pensamento abstrato, permitindo explorar objetos visuais mais realistas para auxiliar a compreensão dos alunos em relação a conceitos científicos, desenvolvendo a análise de informações e a resoluções de problemas.

Na última década, a utilização da RA em processos de aprendizagem tem sido objeto de inúmeras pesquisas, que buscam expor vantagens, tendências, benefícios e desafios (ALZAHRANI, 2020), ou, ainda, verificar como a RA tem sido avaliada na educação (DA SILVA et al., 2019). Embora esses aspectos tenham sido apresentados na literatura, não são observadas revisões sistemáticas relacionadas especificamente ao uso da RA no ensino de Química, tão pouco, à análise de público-alvo e de tópicos que utilizam essa tecnologia, o que criou uma lacuna de conhecimento, necessitando de maior exploração nesta área. Neste contexto, esta Revisão Sistemática de Literatura (RSL) busca contribuir com o preenchimento dessa lacuna, objetivando aprofundar a aplicabilidade e a relevância da Realidade Aumentada no ensino de Química, revelando o público-alvo e os principais tópicos que essa tecnologia é aplicada. 


\section{Materiais e Métodos}

Este estudo caracteriza-se como exploratório e adotou o processo de condução de Revisão Sistemática da Literatura definido por Kitchenham (2004), que objetiva apresentar uma avaliação e interpretação a respeito de um tópico de pesquisa relevante para determinada área, utilizando um método de revisão confiável e passível de auditoria. A execução da RSL foi segmentada em três fases:

a) Planejamento: este trabalho tem como questão de pesquisa principal: "Como a Realidade Aumentada está sendo utilizada no processo de ensino e aprendizagem de Química?". Também tem como propósito contribuir com a análise das seguintes questões secundárias: (i) qual é o público-alvo dos estudos? e (ii) em quais tópicos da Química a Realidade Aumentada é aplicada? Para responder essas questões, optou-se pela análise da literatura internacional somente em língua inglesa. Assim, a seguinte string de pesquisa foi definida: "augmented reality" AND "chemistry" AND "teaching" $A N D$ "learning". Foram recuperados estudos considerando um intervalo de tempo representativo de dez anos, de 2011 a 2020.

b) Condução: a realização desta RSL ocorreu nos meses de janeiro e fevereiro de 2021, sendo que a recuperação dos dados foi realizada nos dias 04 e 05 de janeiro. Por serem renomados no meio acadêmico e científico, foram considerados os repositórios $A C M$ Digital Library, IEEE Xplore Digital Library, ScienceDirect, Scopus, SpringerLink e Web of Science. Foram definidos dois Critérios de Inclusão (CI): o primeiro estabelecendo que o estudo deveria apresentar uma aplicação da RA no processo de ensino aprendizagem de Química e, o segundo, determinando que o estudo deveria, obrigatoriamente, apresentar avaliações com usuários finais. Da mesma forma, foram definidos os seguintes Critérios de Exclusão (CE):

- CE-1: O estudo é literatura cinzenta ou publicação não revisada por pares;

- CE-2: O estudo é secundário, se tratando de uma revisão, de uma meta-análise, de um survey, ou é um trabalho filosófico ou somente teórico;

- CE-3: O estudo é escrito em um idioma diferente do Inglês;

- CE-4: O texto completo do estudo não está disponível;

- CE-5: O estudo não apresenta aplicação da RA no processo de ensino e aprendizagem de Química.

A condução desta RSL foi dividida em três etapas. A primeira envolveu a recuperação dos artigos, por meio da inserção e execução da string de pesquisa nas seis bases de dados e, logo após, a retirada dos artigos duplicados, organizando-os em planilhas (Google Sheets). Na segunda etapa, foram realizadas as leituras do título, resumo e palavras-chave de cada artigo recuperado, confrontando-as com os critérios de inclusão e de exclusão. Este procedimento foi realizado por pares (revisão por pares), ou seja, cada artigo foi analisado individualmente por dois pesquisadores. A terceira e última etapa, consistiu na leitura integral de cada artigo aceito na fase anterior, confrontando-o novamente com os critérios de inclusão e de exclusão. Mantiveram-se os mesmos procedimentos de análise da segunda fase, onde a revisão dos estudos foi realizada por pares, com a decisão de um terceiro pesquisador nos casos de desacordo dos posicionamentos. Por fim, foram aceitos os estudos primários com potencial para gerar respostas à questão de pesquisa. 
c) Relato da Revisão: ao término da primeira etapa da condução, foram encontrados 2161 estudos. Desses, 242 eram duplicados, isto é, retornaram de consultas de mais de um repositório. Assim, ao removê-los, restaram 1919 registros únicos. Na segunda etapa, após a revisão por pares, 1832 registros foram rejeitados e 87 aceitos. Esses foram revisados na terceira etapa, sendo que 44 foram rejeitados e 43 aceitos, os quais consistem no conjunto de estudos primários analisados nesta RSL.

\section{Resultados e Discussão}

A distribuição das publicações no período de 10 anos é apresentada na Figura 1, onde é possível observar uma expressiva ascensão de estudos que utilizam a RA como parte integrante do processo de ensino e aprendizagem de Química. Enquanto os 7 primeiros anos foram pouco representativos, com apenas 7 publicações, os últimos 3 anos mostraram um aumento significativo de estudos, atingindo o maior número em 2020, com 16 artigos.

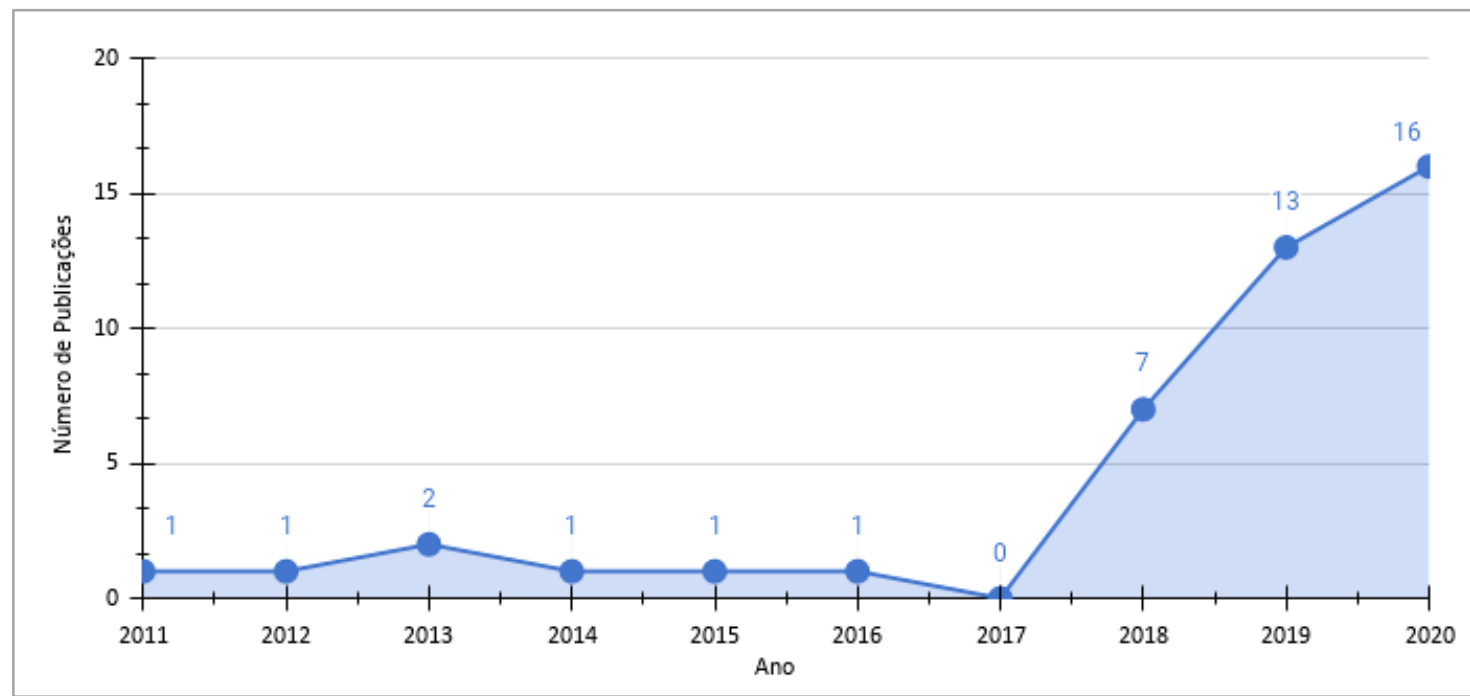

Figura 1 - Disposição de publicações ao longo dos anos.

Esses resultados, aproximam-se dos encontrados na pesquisa de Sirakaya e Alsancak Sirakaya (2020), que indicam um contínuo aumento da aplicação da RA na área educacional. $\mathrm{O}$ que pode ser explicado considerando a crescente disseminação de dispositivos móveis em ambientes educacionais, provocada tanto pela popularização desses equipamentos quanto pela constante evolução tecnológica. Essa evolução, de acordo com Nichele et al. (2020), permitiu que softwares que utilizam recursos de RA fossem adequadamente executados em dispositivos móveis.

\subsection{Qual é o público-alvo dos estudos?}

Como esta RSL realizou buscas em base de dados internacionais, os artigos são provenientes de diferentes países, possuindo um conjunto heterogêneo de informações, como terminologias de níveis, idades de ingresso e período de permanência em cada etapa educacional. Houve, assim, um empenho na tentativa de categorizar o públicoalvo desses estudos, por grau de proximidade da idade dos estudantes (quando mencionadas), ou, ainda, do nível educacional (como "Primary Education", "Lower Secondary Education", "Upper Secondary Education" e "Tertiary"), considerando os 
níveis de ensino propostos pelo Ministério da Educação". A categoria "Múltiplo" foi acrescentada para contemplar um estudo que envolve mais de um tipo de público-alvo, conforme mostra a Figura 2.

Somando-se os 7 estudos (16,28\%) que apresentam o público-alvo como Ensino Fundamental, os 15 estudos que identificam o público-alvo como Ensino Médio $(34,88 \%)$ e 1 estudo caracterizando-o como Múltiplo (2,33\%), tem-se 51,16\% dos estudos reunidos nesses níveis, corroborando a pesquisa de Ibáñez e Delgado-Kloos (2018) que avaliou o uso da RA no apoio à aprendizagem de ciência, tecnologia, engenharia e matemática (STEM). O nível Graduação possui 20 trabalhos, correspondendo a 46,51\% do público-alvo, aproximando-se aos achados de Sirakaya e Alsancak Sirakaya (2018), que buscaram por tendências em estudos educacionais que utilizavam RA e constataram que a maioria das publicações contemplava esse nível educacional.

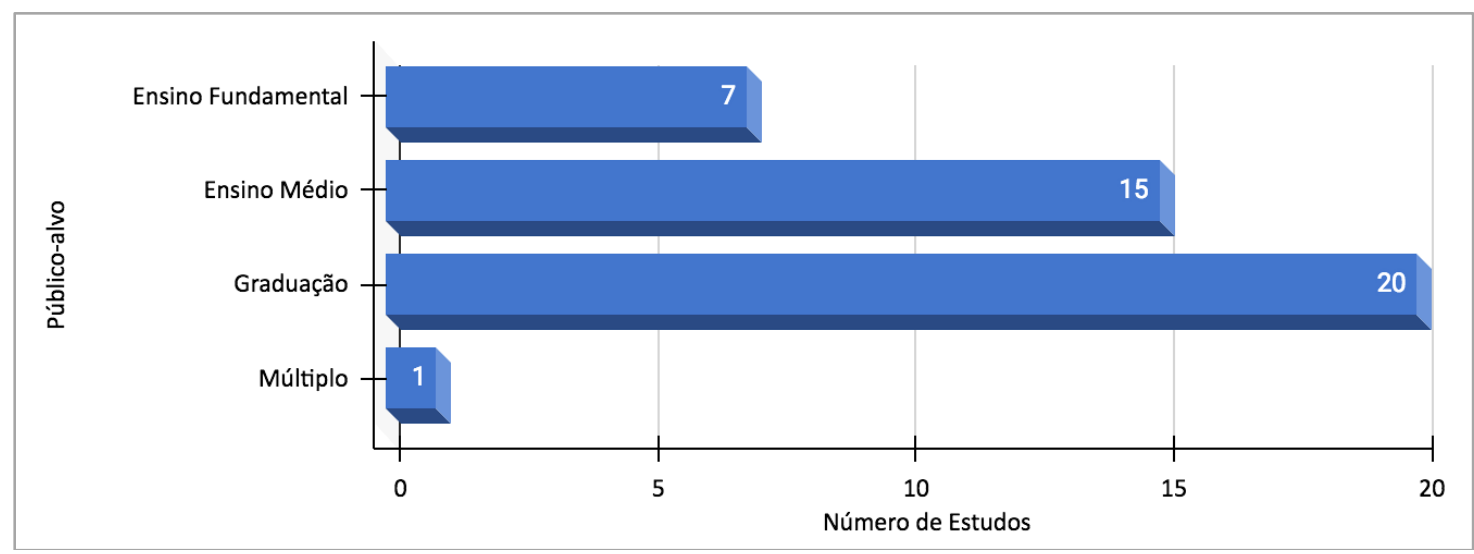

Figura 2 - Público-alvo dos estudos.

Do ponto de vista educacional, a concentração dos estudos na Graduação possivelmente deve-se mais a características da disciplina de Química do que a algum fator envolvendo a tecnologia de RA em si mesma. Como já discutido no início deste texto, a Química é uma área do conhecimento complexa que requer do aprendiz conhecimentos prévios em ciências, noções de física e conceitos sobre matéria. E é justamente na Graduação que esses conhecimentos são exigidos e aprofundados. Talvez, por isso, esse nível de ensino seja um campo fértil para o desenvolvimento e aplicação de sistemas que envolvam a RA.

Por outro lado, observando os números da Figura 2, tem-se uma distribuição significativa de estudos em todos os níveis. Além disso, ao considerar o crescente aumento de publicações nos últimos anos, conforme Figura 1, é possível inferir que a RA, como participante do processo de ensino e aprendizagem de Química, está proporcionando benefícios. Isso pode ser evidenciado por meio de trabalhos, como o de Ewais e Troyer (2019), que contou com um público-alvo de 50 estudantes do Ensino Fundamental, no qual smartphones foram utilizados para realizar experimentos envolvendo a tecnologia de RA para apoiar a aprendizagem de "Ligações Químicas". Os autores perceberam que a RA permitiu o desenvolvimento de atitudes positivas, possibilitando auxiliar os alunos a entender a estrutura e as possíveis interações entre diferentes átomos e moléculas.

Nesse mesmo contexto, outro trabalho de destaque é a pesquisa de Abd Majid e Abd Majid (2018), a qual envolveu o uso de um aplicativo com RA por 25 alunos do

1 https://www.gov.br/mec/pt-br

V. $19 \mathrm{~N}^{\mathrm{o}} 1$, julho, 2021

RENOTE

DOI: https://doi.org/10.22456/1679-1916.118530 
Ensino Médio para apoio à aprendizagem de "Reações Químicas" e a análise de "Estruturas Moleculares". Foi percebido que os estudantes melhoraram seu desempenho e motivação com o uso do aplicativo. Já o estudo de Macariu et al. (2020) envolveu 200 estudantes do Ensino Fundamental e do Ensino Médio que fizeram uso de um aplicativo de RA em seus smartphones e tablets para analisarem "Estruturas Moleculares", "Substâncias" e "Combinações de Elementos". Como benefícios, os autores relataram que tal abordagem aumentou a atratividade, permitiu que alunos retivessem ${ }^{2} \mathrm{com}$ mais facilidade novas informações e melhorou a interação entre estudantes e professores. Por fim, Aw et al. (2020) realizaram um estudo com 87 alunos da Graduação, que utilizaram um aplicativo com RA para trabalhar conceitos relacionados a "Reações Químicas", observação de "Estruturas Moleculares" e "Adição Nucleofílica". Entre as vantagens no uso da RA identificadas, destacou-se o aumento/melhora do interesse/vontade de aprender e aumento da confiança.

\subsection{Em quais tópicos da Química a Realidade Aumentada é aplicada?}

Com a extração de informações dos 43 artigos que compõem o grupo de estudos primários avaliados nesta RSL, obteve-se uma grande diversidade de tópicos da área da Química em que a RA é aplicada. Para apoiar a análise dessas informações, foram consideras apreciações de dois doutores em Química. Inicialmente, houve a intenção de categorizar os estudos. Porém, por dificuldades relativas à granularidade, abrangência de cada tópico e lacunas informacionais sobre eles, foi realizada uma sistematização que buscou conservar as nomenclaturas originalmente utilizadas nos estudos (Tabela 1). Somente em casos específicos, para evitar redundâncias, houve uma padronização conceitual. Por exemplo, tópicos como "Análise de Estruturas Moleculares", "Observação de Estruturas Moleculares" e "Estruturas Moleculares Tridimensionais" foram convencionados como "Estruturas Moleculares".

Tabela 1 - Tópicos da Química em que a RA é aplicada.

\begin{tabular}{l|c|c|l|c|c}
\hline \multicolumn{1}{c|}{ Tópico } & n & \% & \multicolumn{1}{c|}{ Tópico } & n & \% \\
\hline Adição Nucleofílica & 1 & 1,25 & $\begin{array}{l}\text { Medição de } \\
\text { pH/Condutividade }\end{array}$ & 1 & 1,25 \\
\hline Alcanos e Cicloalcanos & 2 & 2,5 & $\begin{array}{l}\text { Mistura e Medição de } \\
\text { Líquidos }\end{array}$ & 1 & 1,25 \\
\hline Bioquímica & 1 & 1,25 & Mudanças Químicas & 1 & 1,25 \\
\hline Célula Eletrolítica & 1 & 1,25 & $\begin{array}{l}\text { Observação/Análise de } \\
\text { Estruturas Moleculares }\end{array}$ & 17 & 21,25 \\
\hline Composição de Matérias & 1 & 1,25 & Precipitação de DNA & 1 & 1,25 \\
\hline Compostos Simples & 1 & 1,25 & Química Analítica & 1 & 1,25 \\
\hline Elementos (Combinações) & 1 & 1,25 & Química Inorgânica & 3 & 3,75 \\
\hline Eletroquímica & 1 & 1,25 & Química Orgânica & 5 & 6,25 \\
\hline Espectroscopia NMR & 1 & 1,25 & Quiralidade Molecular & 2 & 2,5 \\
\hline Estereoquímica & 2 & 2,5 & Reações Químicas & 10 & 12,5 \\
\hline
\end{tabular}

\footnotetext{
2 Apesar de o termo "retenção de informação" muitas vezes não ser bem aceito na área da Educação, optamos por manter sua utilização neste artigo pelo fato de ser essa a terminologia utilizada pelos autores em sua pesquisa.

V. $19 \mathrm{~N}^{\circ} 1$, julho, 2021

RENOTE

DOI: https://doi.org/10.22456/1679-1916.118530
} 


\begin{tabular}{l|c|c|l|c|c}
\hline Estrutura Cristalina & 1 & 1,25 & Reatividade Química & 1 & 1,25 \\
\hline Estruturas Complexas & 1 & 1,25 & $\begin{array}{l}\text { Comp. Orgânicos e } \\
\text { Inorgânicos }\end{array}$ & 1 & 1,25 \\
\hline Experimentos Químicos & 1 & 1,25 & $\begin{array}{l}\text { Representação } \\
\text { Submicroscópica }\end{array}$ & 1 & 1,25 \\
\hline Geometria Molecular & 2 & 2,5 & Segurança Laboratorial & 1 & 1,25 \\
\hline Hibridização Molecular & 1 & 1,25 & $\begin{array}{l}\text { Soluções Eletrolíticas e não } \\
\text { Eletrolíticas }\end{array}$ & 1 & 1,25 \\
\hline Instrumentação & 1 & 1,25 & Tabela Periódica & 4 & 5,0 \\
\hline Ligações Químicas & 9 & 11,25 & Titulação & 1 & 1,25 \\
\hline
\end{tabular}

Foram encontrados um total de 34 tópicos com 80 ocorrências em 43 estudos, o que demonstra a heterogeneidade de assuntos e a complexidade de compreensão desses tópicos, que envolvem propriedades físicas, composições, estruturas e mudanças físicas das matérias (CHANG e CHUNG, 2018). Alguns tópicos estão presentes em mais de um estudo, como "Química Orgânica" e "Reações Químicas", e outros estão presentes em somente um estudo, como "Hibridização Molecular" e "Titulação", por exemplo. Da mesma forma, um trabalho pode abordar mais de um tópico, enquanto outros contemplam apenas um assunto.

O tópico "Estruturas Moleculares" possui 17 ocorrências, o maior número, representando 21,25\%, seguido por "Reações Químicas" com 10 (12,5\%), "Ligações Químicas" com 9 (11,25\%) e "Química Orgânica" com 5 (6,25\%). Assim, somente esses 4 tópicos somam 41 ocorrências $(51,25 \%)$, ou seja, são os tópicos mais frequentes abordados em artigos na área da Química que utilizam a tecnologia de RA em seus processos de ensino e aprendizagem. Já os tópicos "Tabela Periódica" com 4 ocorrências (5\%), "Química Inorgânica" com 3 (3,75\%) e "Alcanos e Cicloalcanos", "Quilaridade Molecular", "Geometria Molecular" e "Estereoquímica" com 2 (2,5\%) cada um, acumulam 15 ocorrências (18,75\%). Com uma representatividade individual menor, de apenas uma ocorrência $(1,25 \%)$, os 24 tópicos restantes somam juntos um índice de $30 \%$.

Os números demonstram que a tecnologia de RA é frequentemente aplicada no ensino de Química, sendo principalmente utilizada para a visualização 3D de estruturas de átomos e moléculas (MACARIU et al., 2020) e de ligações químicas (EWAIS e TROYER, 2019). Possivelmente isso ocorre pelo fato de que conhecer a estrutura 3D das moléculas é essencial para o entendimento de seus comportamentos químicos e de suas propriedades (MAIER e KLINKER, 2013). A percepção de modelos moleculares, juntamente com a compreensão dos processos e estruturas espaciais das moléculas, ainda têm sido causa de dificuldade para os alunos, pois os métodos convencionais de ensino carecem de recursos que possam facilitar o entendimento desses conteúdos (FATEMAH et al., 2020). Isso se deve, principalmente, pelo uso de imagens bidimensionais para ensinar assuntos relacionados a moléculas $3 \mathrm{D}$, o que pode limitar a capacidade de compreensão dos principais elementos visuoespaciais de estruturas moleculares 3D, incluindo percepção de profundidade e senso de escala (WINDAYANI et al., 2020).

Dessa forma, as dificuldades apresentadas pelos alunos em compreender, interpretar e traduzir representações moleculares abstratas e complexas, inerentes a tópicos como "Estruturas Moleculares", "Ligações Químicas" e "Geometria Molecular" podem ser reduzidas com a aplicação da RA em seus respectivos processos de ensino. Essa tecnologia oferece benefícios diferentes em relação ao material de aprendizagem V. $19 \mathrm{~N}^{\circ} 1$, julho, 2021 RENOTE 
clássico (EWAIS e TROYER, 2019), indo além da concretização de conceitos abstratos, abrangendo processos importantes, como a promoção da retenção do conhecimento (CHANG e CHUNG, 2018) e o desenvolvimento de habilidades espaciais (MAIER e KLINKER, 2013).

\section{Considerações Finais}

Esta pesquisa procurou por diálogos entre a Realidade Aumentada e o processo de ensino e aprendizagem em Química, buscando investigar o público-alvo e os tópicos em que essa tecnologia está sendo aplicada. Foram revisados estudos indexados em 6 renomados repositórios internacionais, em um período de 10 anos (de 2011 a 2020). Embora alguns resultados ainda não possam ser considerados conclusivos, certamente possuem a capacidade de serem compreendidos como indícios expressivos, possibilitando suas aplicações em futuras orientações e em novos estudos.

Entre os principais achados acerca do cenário em que a RA é utilizada no processo de ensino e aprendizagem de Química, está o reconhecimento de um progressivo e significativo aumento de publicações nos últimos três anos; a identificação do nível de ensino Graduação (46,51\%) como sendo o público-alvo mais abrangido, apesar de ter sido encontrado um número relevante de estudos nos níveis Ensino Fundamental (16,28\%) e Ensino Médio (34,88\%) e; entre os tópicos da disciplina de Química abordados nas publicações, "Estruturas Moleculares" foi o mais frequente $(21,25 \%)$.

Os resultados podem sugerir que a RA está cada vez mais sendo utilizada no processo de ensino e aprendizagem de Química, e que isso tem relação com a disseminação de dispositivos móveis, provocada, principalmente, pela sua popularização e por eventos de caráter tecnológico, como o aumento do desempenho e da capacidade de processamento gráfico desses dispositivos. Pode-se inferir, do mesmo modo, que a heterogeneidade dos tópicos em Química em que a RA é aplicada é um reflexo natural do aumento do número de pesquisas, o que leva a deduzir que experimentos estão ocorrendo em várias frentes, sobretudo, envolvendo tópicos relacionados às "Estruturas Moleculares".

Somando-se a isso, tem-se um contexto educacional onde, mesmo que alunos do Ensino Fundamental e do Ensino Médico estejam sendo envolvidos em pesquisas, estudantes do nível Graduação são a maioria. Dessa forma, observa-se que para este público é maior a frequência com que estão sendo projetados e implementados diferentes tipos de ferramentas que utilizam a RA como um recurso diferenciado, com o intuito de apoiar o ensino e a aprendizagem de Química e, simultaneamente, evidenciar a colaboração desta tecnologia.

Como lacunas de pesquisa, é possível mencionar a carência de estudos que aprofundem aspectos relacionados tanto ao público-alvo quanto aos tópicos da Química em que a RA é aplicada, destacando a necessidade de que trabalhos futuros sejam dirigidos à identificação desse público, como, idade, sexo, país cujo experimento foi realizado e tipo de instituição de ensino (pública ou privada). Do mesmo modo, considerando a diversidade de países e currículos envolvidos, são necessárias investigações que busquem criar um modelo de categorização de tópicos na esfera curricular ou no âmbito da educação formal. Assim, com maiores detalhes relacionados ao público-alvo e à classificação de tópicos, pode-se aprofundar a compreensão de elementos relacionados ao uso da RA no ensino de Química. 


\section{Referências Bibliográficas}

ABD MAJID, N. A.; ABD MAJID, N. Augmented reality to promote guided discovery learning for STEM learning. Int. J. on Advanced Science, Engineering and Information Technology, v. 8, n. 4-2, p. 1494-1500, 2018.

ADEDOKUN-SHITTU, N. A.; AJANI, A. H.; NUHU, K. M. Augmented reality instructional tool in enhancing geography learners academic performance and retention in Osun state Nigeria. Educ. and Information Technologies, p. 1-13, 2020.

AW, J. K. et al. Interacting with Three-Dimensional Molecular Structures Using an Augmented Reality Mobile App. Journal of Chem. Educ., v. 97, p. 3877-3881. 2020.

ALZAHRANI, N. M. Augmented reality: A systematic review of its benefits and challenges in e-learning contexts. Applied Sciences, v. 10, n. 16, p. 5660, 2020.

AZRINA, K.; JHI-ZHI, L.; DZULKHIFLEE, H. M. The Development of Mobile AR Application to Facilitate High School Students with Various Learning Strategies in Learning History. TURCOMAT, v. 12, n. 3, p. 2199-2204, 2021.

AZUMA, R. T. A survey of augmented reality. Presence: Teleoperators \& Virtual Environments, v. 6, n. 4, p. 355-385, 1997.

BLANCO-NOVOA, O.; FERNANDEZ-CARAMES, T. M.; FRAGA-LAMAS, P.; MONTESINOS, M. A. V. A practical evaluation of commercial industrial augmented reality systems in an industry 4.0 shipyard. IEEE Access, v. 6, p. 8201-8218, 2018.

CAI, S.; LIU, C.; WANG, T.; LIU, E.; LIANG, J. C. Effects of learning physics using Augmented Reality on students' self-efficacy and conceptions of learning. British Journal of Educational Technology, v. 52, n. 1, p. 235-251, 2021.

CELIK, C.; GUVEN, G.; CAKIR, N. K. Integration of mobile augmented reality (MAR) applications into biology laboratory: Anatomic structure of the heart. Research in Learning Technology, v. 28, 2020.

CHANG, R. C.; CHUNG, L. Y. Integrating augmented reality technology into subject teaching: The implementation of an elementary science curriculum. In: International Conference on Frontier Computing. Springer, Singapore, 2016. p. 187-195.

DA SILVA, M. M. O.; TEIXEIRA, J. M. X.; CAVALCANTE, P. S.; TEICHRIEB, V. Perspectives on how to evaluate augmented reality technology tools for education: a systematic review. Journal of the Brazilian Computer Society, v. 25, p. 1-18, 2019.

EWAIS, A.; TROYER, O. A usability and acceptance evaluation of the use of augmented reality for learning atoms and molecules reaction by primary school female students in Palestine. Journal of Educ. Comp. Res., v. 57, n. 7, p. 1643-1670, 2019.

FATEMAH, A.; RASOOL, S.; HABIB, U. Interactive 3D Visualization of Chemical Structure Diagrams Embedded in Text to Aid Spatial Learning Process of Students. Journal of Chemical Education, v. 97, n. 4, p. 992-1000, 2020. 
GSAXNER, C.; PEPE, A.; LI, J.; IBRAHIMPASIC, U. Augmented Reality for Head and Neck Carcinoma Imaging: Description and Feasibility of an Instant Calibration, Markerless Approach. Comp. Methods and Prog. in Biom., v. 200, p. 105854, 2021.

HAN, S.; YOON, J. H.; KWON, J. Impact of Experiential Value of Augmented Reality: The Context of Heritage Tourism. Sustainability, v. 13, n. 8, p. 4147, 2021.

HANAFI, A.; ELAACHAK, L.; BOUHORMA, M. Augmented Reality Application in Laboratories and Learning Procedures. In: The Proceedings of the Third International Conf. on Smart City Applications. Springer, Cham, 2019. p. 157-167.

IBÁÑEZ, M. B.; DELGADO-KLOOS, C. Augmented reality for STEM learning: A systematic review. Computers \& Education, v. 123, p. 109-123, 2018.

KERR, J.; LAWSON, G. Augmented reality in design education: landscape architecture studies as AR experience. Int. J. of Art \& Design Education, v. 39, n. 1, p. 6-21, 2020.

KITCHENHAM, B. Procedures for performing systematic reviews. Keele, UK, Keele University, 33, p. 1-26. 2004.

KONONOVA, N.; SHIRYAEVA, N.; OBLASOVA, I. The Use of Augmented Reality Technology in the Educational Process. In: CEUR Workshop Proceedings. 2019.

MAIER, P.; KLINKER, G. Augmented chemical reactions: An augmented reality tool to support chemistry teaching. In 2nd Experiment@ Int. Conf., p. 164-165, 2013.

MACARIU, C.; IFTENE, A.; GÎFU, D. Learn Chemistry with Augmented Reality. Procedia Computer Science, v. 176, p. 2133-2142, 2020.

NICHELE, A. G.; DO CANTO, L. Z.; DA SILVA F. N. Augmented Reality: Apps for Teaching and Learning Chemistry. In 14th International Technology, Education and Development Conference, p. 7650-7655, 2020.

ÖNAL, N. T.; ÖNAL, N. The effect of augmented reality on the astronomy achievement and interest level of gifted students. Education and Information Technologies, p. 1-27, 2021.

PRIBEANU, C.; BALOG, A.; IORDACHE, D. D. Measuring the perceived quality of an AR-based learning application: a multidimensional model. Interactive Learning Environments, v. 25, n. 4, p. 482-495, 2017.

SIRAKAYA, M.; ALSANCAK SIRAKAYA, D. Trends in educational augmented reality studies: A systematic review. Malaysian Online Journal of Educational Technology, v. 6, n. 2, p. 60-74, 2018.

SIRAKAYA, M.; ALSANCAK SIRAKAYA, D. Augmented reality in STEM education: A systematic review. Interactive Learning Environments, p. 1-14, 2020.

SYAWALUDIN, A.; GUNARHADI, G.; RINTAYATI, P. Enhancing elementary school students' abstract reasoning in science learning through augmented reality-based interactive multimedia. Journal Pendidikan IPA Indonesia, p. 288-297, 2019. 
VELÁZQUEZ, F. D. C.; MÉNDEZ, G. M. Application in Augmented Reality for Learning Mathematical Functions: A Study for the Development of Spatial Intelligence in Secondary Education Students. Mathematics, v. 9, n. 4, p. 369, 2021.

WINDAYANI, N.; IRWANSYAH, F. S.; ASYIAH, E. N. Making Augmented Reality Learning Media in Conformation of Alkane and Cycloalkane Concepts. In 2019 IEEE 5th International Conference on Wireless and Telematics, p. 1-5, 2019.

ZHANG, Z.; LI, Z.; HAN, M.; SU, Z.; LI, W.; PAN, Z. An augmented reality-based multimedia environment for experimental education. Multimedia Tools and Applications, v. 80, n. 1, p. 575-590, 2021. 